\title{
Flow and Heat Transfer of Casson Fluid from a horizontal Circular Cylinder with Partial Slip in non-Darcy porous Medium
}

\author{
Ramachandra Prasad V ${ }^{1 *}$, Subba Rao $\mathrm{A}^{1}$ and Anwar Bég O²
}

${ }^{1}$ Department of Mathematics, Madanapalle Institute of Technology and Science, Madanapalle-517325, India

${ }^{2}$ Aerospace Engineering, Sheffield Hallam University, Sheaf St, Sheffield, S11WB, UK

\begin{abstract}
In the Present study, the steady flow and heat transfer of Casson fluid from a permeable horizontal cylinder in the presence of slip condition in a non-Darcy porous medium is analyzed. The cylinder surface is maintained at a constant temperature. The boundary layer conservation equations, which are parabolic in nature, are normalized into non-similar form and then solved numerically with the well-tested, efficient, implicit, stable Keller-box finite-difference scheme. Increasing the velocity slip parameter is found to decrease the velocity and boundary layer thickness and increases the temperature and the boundary layer thickness. The velocity decreases with the increase the nonDarcy parameter and is found to increase the temperature. The velocity increases with the increase the Casson fluid parameter and is found to decrease the temperature. The Skin-friction coefficient and the local Nusselt number are found to decrease with the increase in velocity and thermal slip parameters respectively.
\end{abstract}

Keywords: Casson fluid; Slip condition; Heat transfer; Non-Darcy porous medium yield stress; Keller-box numerical method; Skin friction; Nusselt number; Boundary layers; Chocolate food processing

\section{Nomenclature}

$a$ : radius of the cylinder

$C_{f}$ : skin friction coefficient

$D a:$ Darcy parameter

$\Gamma$ : inertia coefficient

$S_{f}:$ non-dimensional velocity slip parameter

$S_{T}$ : non-dimensional thermal slip parameter

$f$ : non-dimensional steam function

$g$ : acceleration due to gravity

$G r:$ Grashof number

$K$ : thermal diffusivity

$N_{0}$ : velocity slip factor

$K_{0}$ : thermal slip factor

$N u$ : Local Nusselt number

$\operatorname{Pr}$ : Prandtl number

$T$ : temperature

$\mathrm{u}, \mathrm{v}$ : non-dimensional velocity components along the $\mathrm{x}$ - and $\mathrm{y}$ directions, respectively

$\mathrm{x}, \mathrm{y}$ : non-dimensional Cartesian coordinates along the surface of the cylinder and normal to it, respectively

\section{Greek Symbols}
$\alpha$ : thermal diffusivity
$\Omega$ : the coefficients of thermal expansion
$\beta$ : the non-Newtonian Casson parameter
$\Phi$ : the azimuthal coordinate
$\eta$ : the dimensionless radial coordinate
$\mu$ : dynamic viscosity
$v$ : kinematic viscosity
$\theta$ : non-dimensional temperature
$\rho:$ density
$\xi$ : the dimensionless tangential coordinate
$\psi:$ dimensionless stream function

\section{Subscripts}

$$
\mathrm{w} \text { : conditions on the wall }
$$

$\infty$ : free stream conditions

\section{Introduction}

Non-Newtonian transport phenomena arise in many branches of chemical and materials processing engineering. Such fluids exhibit shear-stress-strain relationships which diverge significantly from the Newtonian (Navier-Stokes) model. Most non-Newtonian models involve some form of modification to the momentum conservation equations. These include power-law, thixotropic and viscoelastic fluids [1]. Such rheological models however cannot simulate the micro

*Corresponding author: V. Ramachandra Prasad, Department of Mathematics, Madanapalle Institute of Technology and Science, Madanapalle-517325, India, Tel: +91-9160020785, E-mail: rcpmaths@gmail.com

Received March 21, 2013; Accepted May 08, 2013; Published May 17, 2013

Citation: Ramachandra Prasad V, Subba Rao A, Anwar Bég O (2013) Flow and Heat Transfer of Casson Fluid from a horizontal Circular Cylinder with Partial Slip in non-Darcy porous Medium. J Appl Computat Math 2: 127. doi:10.4172/21689679.1000127

Copyright: @ 2013 Ramachandra Prasad V, et al. This is an open-access article distributed under the terms of the Creative Commons Attribution License, which permits unrestricted use, distribution, and reproduction in any medium, provided the original author and source are credited. 
structural characteristics of many important liquids including polymer suspensions, liquid crystal melts, physiological fluids, contaminated lubricants, etc.

The steady flow of non-Newtonian fluids in the presence of heat transfer is an important research area due to its wide use in food processing, power engineering, and petroleum production and in many industries for example polymers melt and polymer solutions employed in the plastic processing. Several fluids in chemical engineering, multiphase mixtures, pharmaceutical formulations, china clay and coal in water, paints, synthetic lubricants, salvia, synovial fluid, jams, soups, jellies, marmalades, sewage sludge etc are non-Newtonian. The constitutive relations for these kinds of fluids give rise to more complex and higher order equations than the Navier-Stokes equations. Considerable progress even through has been made on the topic by using different models of non-Newtonian fluids [2-11].

Transport processes in porous media can involve fluid, heat and mass transfer in single or multi-phase scenarios. Such flows with and without buoyancy effects arise frequently in many branches of chemical engineering and owing to their viscous-dominated nature are generally simulated using the Darcy model. Applications of such flows include chip-based micro fluidic chromatographic separation devices [12], dissolution of masses buried in a packed bed [13], heat transfer in radon saturating permeable regimes [14], flows in ceramic filter components of integrated gasification combined cycles (IGCC) [15], separation of carbon dioxide from the gas phase with aqueous absorbents (water and di-ethanolamine solution) in micro porous hollow fibre membrane modules [16], and monolithic adsorbent flows consisting of micro-porous zeolite particles embedded in a polyamide matrix [17]. Porous media flow simulations are also critical in convective processes in hygroscopic materials [18], electro remediation in soil decontamination technique wherein an electric field applied to a porous medium generates the migration of ionic species in solution [19], reactive transport in tubular porous media reactors [20], perfusive bed flows [21], situ gelation of biopolymers in porous media which arise in petroleum recovery and in subsurface heavy metal stabilization [22].

Previous studies indicate that not much has been presented yet regarding Casson fluid. This model [23-25] in fact is a plastic fluid that exhibits shear thinning characteristics and that quantifies yield stress and high shear viscosity. Casson fluid model is reduced to a Newtonian fluid at very high wall shear stresses, when wall stress is much greater than yield stress. This fluid has good approximations for many substances such as biological materials, foams, molten chocolate, cosmetics, nail polish, some particulate suspensions etc. The boundary layer behaviour of viscoelastic fluid has technical applications in engineering such as glass fiber, paper production, manufacture of foods, the aerodynamic extrusion of plastic sheets, the polymer extrusion in a melt spinning process and many others.

The objective of the present paper is to investigate the steady boundary-layer flow and heat transfer of Casson fluid past a horizontal cylinder in a non-Darcy porous medium. Mathematical modelling through equations of continuity and motion leads to a nonlinear differential equation even after employing the boundary layer assumptions. The velocity and thermal slip conditions along with conservation law of mass, momentum and energy completes the problems formulation for velocity components and temperature. The considered slip conditions especially are important in the nonNewtonian fluids such as polymer melts which often exhibit wall slip. It has been experimentally verified that fluid possesses non-continuum features such as slip flow when the molecular mean free path length of fluid is comparable to the distance between the plates as in Nano channels/micro channels [26].

\section{Mathematical Analysis}

The steady, laminar, two-dimensional, viscous, incompressible, buoyancy-driven convection heat transfer flow from a horizontal permeable circular cylinder embedded in a Casson non-Newtonian fluid. Figure 1 shows the flow model and physical coordinate system. The $\mathrm{x}$-coordinate is measured along the circumference of the horizontal cylinder from the lowest point and the y-coordinate is measured normal to the surface, with ' $a$ ' denoting the radius of the horizontal cylinder. $\Phi=x / a$ is the angle of the $y$-axis with respect to the vertical $(0 \leq \Phi \leq \pi)$. The gravitational acceleration, ' $g$ ' acts downwards. Both the horizontal cylinder and the fluid are maintained initially at the same temperature. Instantaneously they are raised to a temperature $T_{w}>T_{\infty}$, the ambient temperature of the fluid which remains unchanged.

We also assume the rheological equation of Casson fluid, reported by Mustafa et al. [27] and recently Nadeem et.al [28] is:

$$
\tau^{1 / n}=\tau_{0}^{1 / n}+\mu \dot{\gamma}^{1 / n}
$$

or

$$
\tau_{i j}=\left[\mu_{B}+\left(\frac{P_{y}}{\sqrt{2 \pi}}\right)^{1 / n}\right]^{n} 2 e_{i j}
$$

Where $\mu$ is the dynamic viscosity, $\mu_{B}$ the plastic dynamic viscosity of non-Newtonian fluid, $\pi=e_{i j} e_{i j}$ and $e_{i j}$ is the $(i, j)^{t h}$ component of deformation rate, $\pi$ denotes the product of the component of deformation rate with itself, $\pi_{c}$ shows a critical value of this product based on the non-Newtonian model, and $p_{y}$ the yield stress of fluid. We consider a steady state flow. An anonymous referee has suggested considering the value of $n=1$. However, in many applications this value is $\mathrm{n} \gg 1$.

In line with the approach of Yih [29] and introducing the boundary layer approximations, the governing conservation equations can be written as follows:

$$
\begin{aligned}
& \frac{\partial u}{\partial x}+\frac{\partial v}{\partial y}=0 \\
& u \frac{\partial u}{\partial x}+v \frac{\partial u}{\partial y}=v\left(1+\frac{1}{\beta}\right) \frac{\partial^{2} u}{\partial y^{2}}+g \Omega\left(T-T_{\infty}\right) \sin \left(\frac{x}{a}\right)-\frac{v}{K} u-\Gamma u^{2}
\end{aligned}
$$

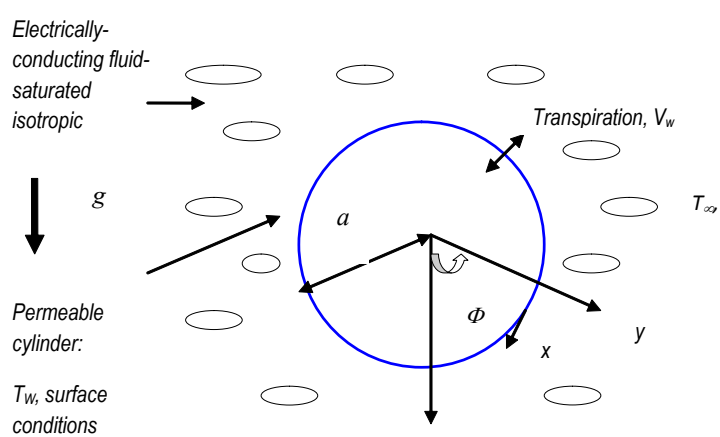

Figure 1: Physical Model and Coordinate System. 


$$
u \frac{\partial T}{\partial x}+v \frac{\partial T}{\partial y}=\alpha \frac{\partial^{2} T}{\partial y^{2}}
$$

where $u$ and $v$ are the velocity components in the $x$ - and $y$ directions respectively, $v$-the kinematic viscosity of the conducting fluid, $\beta$-is the non-Newtonian Casson parameter, $\alpha$-the thermal diffusivity, $T$-the temperature, $K$ and $\Gamma$-the respective permeability and the inertia coefficient of the porous medium, $\Omega$ is the coefficients of thermal expansion, $T_{\infty}$-the free stream temperature.

The boundary conditions are prescribed at the cylinder surface and the edge of the boundary layer regime, respectively as follows:

$$
\text { At } y=0, \quad u=N_{0}\left(1+\frac{1}{\beta}\right) \frac{\partial u}{\partial y}, \quad v=-V_{w}, \quad T=T_{w}+K_{0} \frac{\partial T}{\partial y}
$$$$
\text { As } y \rightarrow \infty, \quad u \rightarrow 0, T \rightarrow T_{\infty}
$$

Where $N_{0}$ the velocity is slip factor and $K_{0}$ is the thermal slip factor. For $N_{0}=0=K_{0}$, one can recover the no-slip case.

The stream function $\psi$ is defined by $u=\partial \psi / \partial y$ and $v=-\partial \psi / \partial x$, and therefore, the continuity equation is automatically satisfied. In order to write the governing equations and the boundary conditions in dimensionless form, the following non-dimensional quantities are introduced.

$$
\left\{\begin{array}{lcc}
\xi=\frac{x}{a}, \quad \eta=\frac{y}{a} \sqrt[4]{G r}, & f(\xi, \eta)=\frac{\psi}{v \xi \sqrt[4]{G r}}, & \operatorname{Pr}=\frac{v}{\alpha} \\
\theta(\xi, \eta)=\frac{T-T_{\infty}}{T_{w}-T_{\infty}}, \quad G r=\frac{g \Omega\left(T_{w}-T_{\infty}\right) a^{3}}{v^{2}}, & \beta=\mu_{B} \frac{\sqrt{2 \pi_{c}}}{p_{y}} \\
\Lambda=\Gamma a, \quad & D a=\frac{K}{a^{2}}, \quad f_{w}=-\frac{V_{w} a}{v \sqrt[4]{G r}} &
\end{array}\right.
$$

In view of non-dimensional quantities (7), Equations (3)-(5) reduce to the following coupled, nonlinear, dimensionless partial differential equations for momentum and energy for the regime

$$
\begin{aligned}
& \left(1+\frac{1}{\beta}\right) f^{\prime \prime \prime}+f f^{\prime \prime}-(1+\xi \Lambda) f^{\prime^{2}}-\left(\frac{1}{D a G r^{1 / 2}}\right) f^{\prime}+\frac{\sin \xi}{\xi} \theta=\xi\left(f^{\prime} \frac{\partial f^{\prime}}{\partial \xi}-f^{\prime \prime} \frac{\partial f}{\partial \xi}\right) \\
& \frac{\theta^{\prime \prime}}{\operatorname{Pr}}+f \theta^{\prime}=\xi\left(f^{\prime} \frac{\partial \theta}{\partial \xi}-\theta^{\prime} \frac{\partial f}{\partial \xi}\right)
\end{aligned}
$$

The transformed dimensionless boundary conditions are:

$$
\begin{aligned}
& \text { At } \eta=0, \quad f=S, \quad f^{\prime}=\left(1+\frac{1}{\beta}\right) S_{f} f^{\prime \prime}(0), \quad \theta=1+S_{T} \theta^{\prime}(0) \\
& \text { As } \eta \rightarrow \infty, \quad f^{\prime} \rightarrow 0, \quad \theta \rightarrow 0
\end{aligned}
$$

In the above equations, the primes denote the differentiation with respect to $\eta$, the dimensionless radial coordinate, and $\xi$ is the dimensionless tangential coordinate, $\Lambda$-the local inertia coefficient (Forchheimer parameter), $D a$ - the Darcy parameter and $G r$ is the Grashof (free convection) parameter. $\operatorname{Pr}=\frac{v}{\alpha}$ is the Prandtl number, $S_{f}=\frac{N_{0} G r^{1 / 4}}{a}$ and $S_{T}=\frac{K_{0} G r^{1 / 4}}{a}$ are the non-dimensional velocity and thermal slip parameters respectively and $f_{w}$ is the blowing/suction parameter. $f_{w}<0$ for $V_{w}>0$ (the case of blowing), and $f_{w}>0$ for $V_{w}<0$ (the case of suction). Of course the special case of a solid cylinder surface corresponds to $f_{w}=0$.

The engineering design quantities of physical interest include the skin-friction coefficient and Nusselt number, which are given by:

$$
\begin{aligned}
& C_{f} G r^{-3 / 4}=\left(1+\frac{1}{\beta}\right) \xi f^{\prime \prime}(0) \\
& \frac{N u}{\sqrt[4]{G r}}=-\theta^{\prime}(0)
\end{aligned}
$$

\section{Numerical Solution}

In this study the efficient Keller-Box implicit difference method has been employed to solve the general flow model defined by equations (8)-(9) with boundary conditions (10). Therefore a more detailed exposition is presented here. This method, originally developed for low speed aerodynamic boundary layers by Keller [30], and has been employed in a diverse range of coupled heat transfer problems. These include Ramachandra Prasad et al. [31-32] and Beg et al. [33].

Essentially 4 phases are central to the Keller Box Scheme.

These are:

a. Reduction of the $\mathrm{N}^{\text {th }}$ order partial differential equation system to $\mathrm{N}$ first order equations

b. Finite Difference Discretization

c. Quasilinearization of Non-Linear Keller Algebraic Equations

d. Block-tridiagonal Elimination of Linear Keller Algebraic Equations

Phase a: Reduction of the $\mathrm{N}^{\text {th }}$ order partial differential equation system to $\mathrm{N}$ first order equations

Equations (8)-(9) subject to the boundary conditions (10) are first written as a system of first-order equations. For this purpose, we reset Equations (8)-(9) as a set of simultaneous equations by introducing the new variables $\mathrm{u}$, vandt:

$$
\begin{aligned}
& f^{\prime}=u \\
& f^{\prime \prime}=v \\
& \theta^{\prime}=t \\
& \left(1+\frac{1}{\beta}\right) v^{\prime}+f v-(1+\xi \Lambda) u^{2}-\left(\frac{1}{D a G r^{1 / 2}}\right) u+\frac{\sin \xi}{\xi} s=\xi\left(u \frac{\partial u}{\partial \xi}-v \frac{\partial f}{\partial \xi}\right) \\
& \frac{1}{\operatorname{Pr}} t^{\prime}+f t=\xi\left(u \frac{\partial s}{\partial \xi}-t \frac{\partial f}{\partial \xi}\right)
\end{aligned}
$$

Where primes denote differentiation with respect to $\eta$. In terms of the dependent variables, the boundary conditions become:

$$
\begin{array}{ll}
\text { At } & \eta=0: \quad u=\left(1+\frac{1}{\beta}\right) f^{\prime \prime}(0), \quad f=f_{w}, \quad s=1 \\
\text { As } & \eta \rightarrow \infty: \quad u \rightarrow 0, \quad s \rightarrow 0
\end{array}
$$

\section{Phase b: Finite difference discretization}

A two dimensional computational grid is imposed on the $\xi_{-\eta}$ plane as sketched in Figure 2. The stepping process is defined by:

$$
\begin{aligned}
& \eta_{0}=0, \quad \eta_{j}=\eta_{j-1}+h_{j}, \quad j=1,2, \ldots, J, \quad \eta_{J} \equiv \eta_{\infty} \\
& \xi^{0}=0, \quad \xi^{n}=\xi^{n-1}+k_{n}, \quad n=1,2, \ldots, N
\end{aligned}
$$




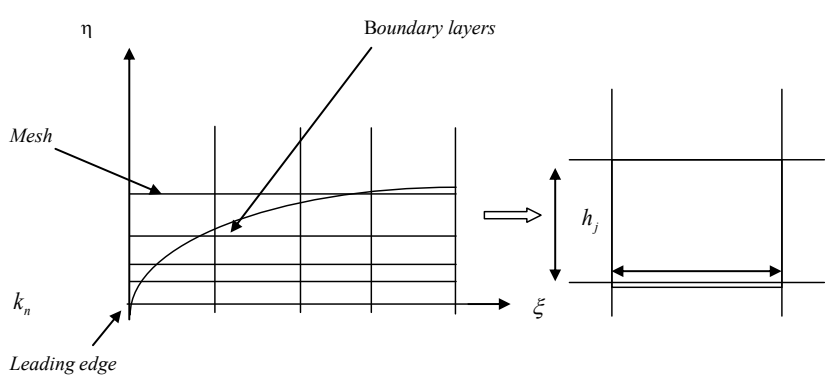

Figure 2: Grid Meshing and a Keller Box Computational Cell.

Where $k_{n}$ and $h_{j}$ denote the step distances in $\xi$ and $\eta$ directions respectively.

If $g_{j}^{n}$ denotes the value of any variable at $\left(\eta_{j}, \xi^{n}\right)$, then the variables and derivatives of equations. $(13)-(17)$ at $\left(\eta_{j-1 / 2}, \xi^{n-1 / 2}\right)$ are replaced by

$$
\begin{aligned}
& g_{j-1 / 2}^{n-1 / 2}=\frac{1}{4}\left(g_{j}^{n}+g_{j-1}^{n}+g_{j}^{n-1}+g_{j-1}^{n-1}\right), \\
& \left(\frac{\partial g}{\partial \eta}\right)_{j-1 / 2}^{n-1 / 2}=\frac{1}{2 h_{j}}\left(g_{j}^{n}-g_{j-1}^{n}+g_{j}^{n-1}-g_{j-1}^{n-1}\right), \\
& \left(\frac{\partial g}{\partial \xi}\right)_{j-1 / 2}^{n-1 / 2}=\frac{1}{2 k^{n}}\left(g_{j}^{n}-g_{j-1}^{n}+g_{j}^{n-1}-g_{j-1}^{n-1}\right),
\end{aligned}
$$

We now show the finite-difference approximation of equations. (13) - (17) for the mid-point $\left(\eta_{j-1 / 2}, \xi^{n}\right)$, below

$$
\begin{aligned}
& h_{j}^{-1}\left(f_{j}^{n}-f_{j-1}^{n}\right)=u_{j-1 / 2}^{n}, \\
& h_{j}^{-1}\left(u_{j}^{n}-u_{j-1}^{n}\right)=v_{j-1 / 2}^{n},
\end{aligned}
$$

$\left(1+\frac{1}{\beta}\right)\left(v_{j}-v_{j-1}\right)+\frac{(1+\alpha) h_{j}}{4}\left[\left(f_{j}+f_{j-1}\right)\left(v_{j}+v_{j-1}\right)\right]-\frac{h_{j}}{4}(1+\alpha+\xi \Lambda)\left(u_{j}+u_{j-1}\right)^{2}$

$$
-\left(\frac{1}{D a G r^{1 / 2}}\right) \frac{h_{j}}{2}\left(u_{j}+u_{j-1}\right)-\frac{\alpha h_{j}}{2} f_{j-1 / 2}^{n-1}\left(v_{j}+v_{j-1}\right)
$$$$
+\frac{\alpha h_{j}}{2} v_{j-1 / 2}^{n-1}\left(f_{j}+f_{j-1}\right)+\frac{B h_{j}}{2}\left(s_{j}+s_{j-1}\right)=\left[R_{1}\right]_{j-1 / 2}^{n-1}
$$

$h_{j}^{-1}\left(\theta_{j}^{n}-\theta_{j-1}^{n}\right)=t_{j-1 / 2}^{n}$

$$
\begin{aligned}
\frac{1}{\operatorname{Pr}}\left(t_{j}-t_{j-1}\right)+\frac{(1+\alpha) h_{j}}{4}\left[\left(f_{j}+f_{j-1}\right)\left(t_{j}+t_{j-1}\right)\right]-\frac{\alpha h_{j}}{4}\left[\left(u_{j}+u_{j-1}\right)\left(s_{j}+s_{j-1}\right)\right] \\
+\frac{\alpha h_{j}}{2} s_{j-1 / 2}^{n-1}\left(u_{j}+u_{j-1}\right)-\frac{\alpha h_{j}}{2} u_{j-1 / 2}^{n-1}\left(s_{j}+s_{j-1}\right)-\frac{\alpha h_{j}}{2} f_{j-1 / 2}^{n-1}\left(t_{j}+t_{j-1}\right) \\
+\frac{\alpha h_{j}}{2} t_{j-1 / 2}^{n-1}\left(f_{j}+f_{j-1}\right)=\left[R_{2}\right]_{j-1 / 2}^{n-1}
\end{aligned}
$$

Where we have used the abbreviations

$$
\begin{gathered}
\alpha=\frac{\xi^{n-1 / 2}}{k_{n}}, B=\frac{\sin \left(\xi^{n-1 / 2}\right)}{\xi^{n-1 / 2}} \\
{\left[R_{1}\right]_{j-1 / 2}^{n-1}=-h_{j}\left[\left(1+\frac{1}{\beta}\right)\left(\frac{v_{j}-v_{j-1}}{h_{j}}\right)+(1-\alpha)\left(f_{j-1 / 2} v_{j-1 / 2}\right)-(1-\alpha+\xi \Lambda)\left(u_{j-1 / 2}\right)^{2}\right.} \\
\left.+\left(\frac{1}{D a G r^{1 / 2}}\right) u+B\left(s_{j-1 / 2}\right)\right]
\end{gathered}
$$

$$
\left[R_{2}\right]_{j-1 / 2}^{n-1}=-h_{j}\left[\frac{1}{\operatorname{Pr}}\left(\frac{t_{j}-t_{j-1}}{h_{j}}\right)+(1-\alpha)\left(f_{j-1 / 2} t_{j-1 / 2}\right)+\alpha\left(u_{j-1 / 2} s_{j-1 / 2}\right)\right]
$$

The boundary conditions are

$$
f_{0}^{n}=u_{0}^{n}=0, \quad \theta_{0}^{n}=1, \quad u_{J}^{n}=0, \quad \theta_{J}^{n}=0
$$

Phase c: Quasilinearization of non-linear Keller algebraic equations

If we assume $f_{j}^{n-1}, u_{j}^{n-1}, v_{j}^{n-1}, s_{j}^{n-1}, t_{j}^{n-1}$ to be known for $0 \leq j \leq J$, Equations (24)-(28) are a system of $5 J+5$ equations for the solution of $5 J+5$ unknowns $f_{j}^{n}, u_{j}^{n}, v_{j}^{n}, s_{j}^{n}, t_{j}^{n}, j=0,1,2,3 \ldots J$. This non-linear system of algebraic equations is linearized by means of Newton's method as explained in [31].

Phase d: Block-tridiagonal elimination of linear Keller algebraic equations

The linear system (24)-(28) can now be solved by the blockelimination method. The linearized difference equations of the system have a block-tridiagonal structure. Commonly, the block-tridiagonal structure consists of variables or constants, but here, an interesting feature can be observed that is, for the Keller-box method, it consists of block matrices. The complete linearized system is formulated as block matrix system, where each element in the coefficient matrix is a matrix itself. Then, this system is solved using the efficient Keller-box method as developed by Cebeci and Bradshaw [34]. The numerical results are affected by the number of mesh points in both directions. After some trials in the $\eta$-direction a larger number of mesh points are selected whereas in the direction significantly less mesh points are utilized. $\eta_{\max }$ has been set at 15 and this defines an adequately large value at which the prescribed boundary conditions are satisfied. $\xi_{\max }$ is set at 3.0 for this flow domain.

\section{Results and Discussions}

Comprehensive solutions have been obtained and are presented in figures 3-24. The numerical problem comprises 2 independent variables $(\xi, \eta), 2$ dependent fluid dynamic variables $(f, \theta)$ and 8thermo physical and bodyforce control parameters $\operatorname{Pr}, \mathrm{S}_{\mathrm{f}}, \mathrm{S}_{\mathrm{T}}, \beta, f_{w}, \xi, D a, \Lambda$.Inthepresent computations, the following default parameters are prescribed (unless otherwise stated): $\operatorname{Pr}=10, S_{f}=0.5, S_{T}=1.0, \beta=1.0, f_{w}=0.5, \xi=1.0$

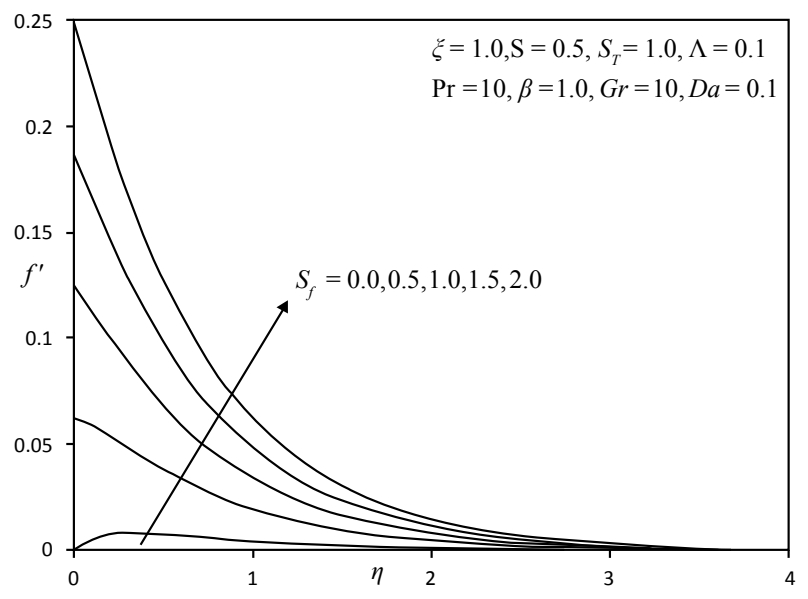

Figure 3: Influence of $S_{f}$ on velocity profiles. 
Citation: Ramachandra Prasad V, Subba Rao A, Anwar Bég O (2013) Flow and Heat Transfer of Casson Fluid from a horizontal Circular Cylinder with Partial Slip in non-Darcy porous Medium. J Appl Computat Math 2: 127. doi:10.4172/2168-9679.1000127

Page 5 of 12

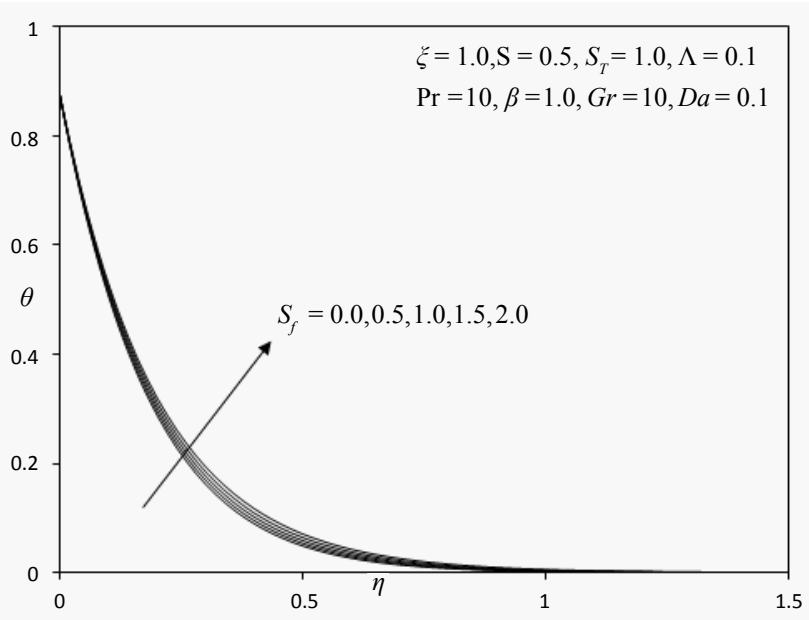

Figure 4: Influence of $S_{f}$ on temperature profiles

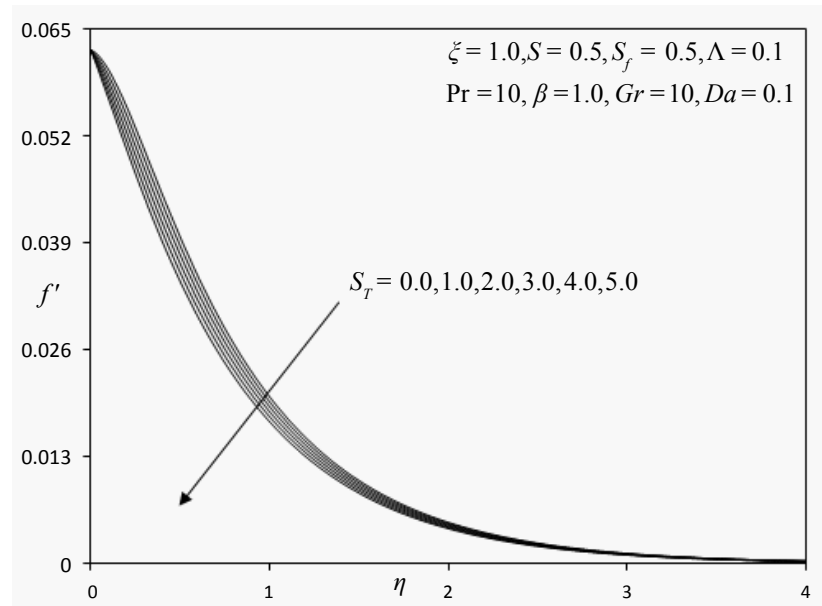

Figure 5: Influence of $\mathrm{S}_{\mathrm{T}}$ on velocity profiles

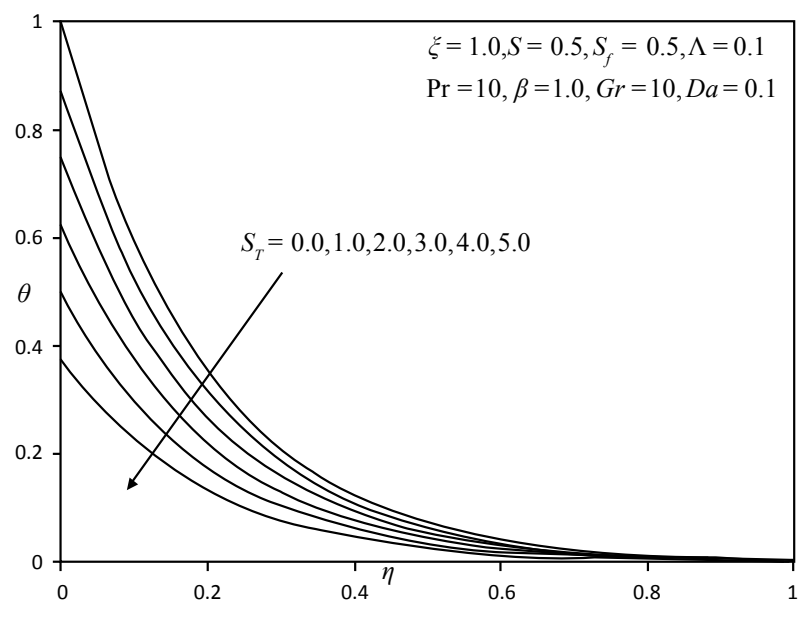

Figure 6: Influence of $\mathrm{S}_{\mathrm{T}}$ on temperature profiles.

$D a=0.1$ and $\Lambda=0.1$. In addition we also consider the effect of stream wise coordinate location on flow dynamics. The value of the parameter

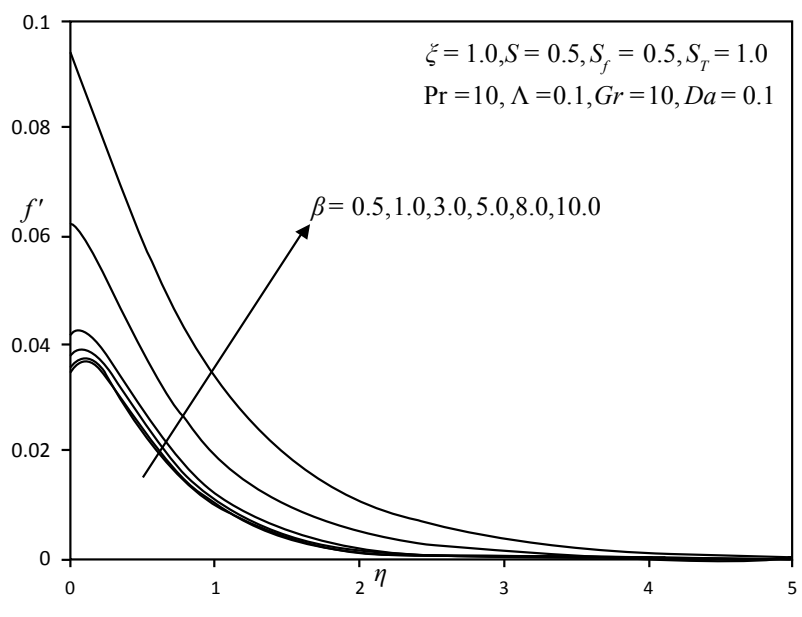

Figure 7: Influence of $\beta$ on velocity profiles.

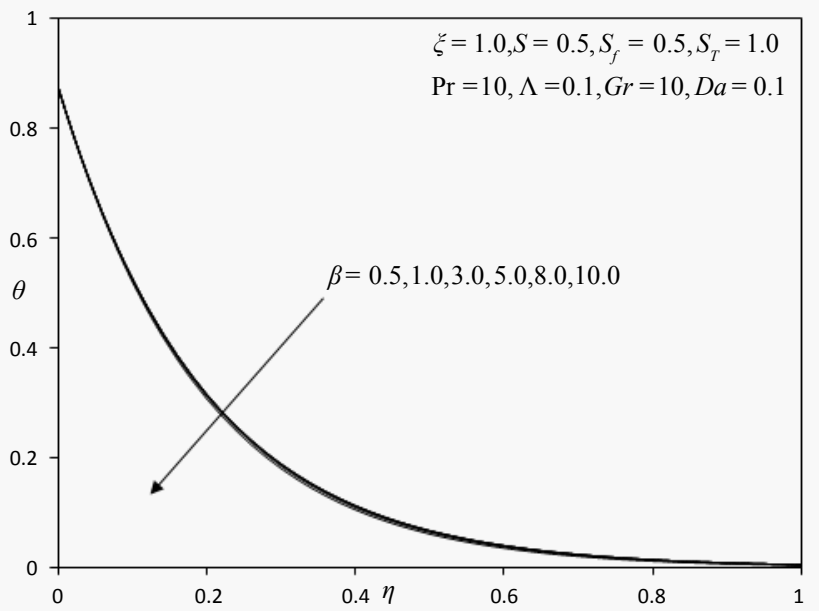

Figure 8: Influence of $\beta$ on temperature profiles.

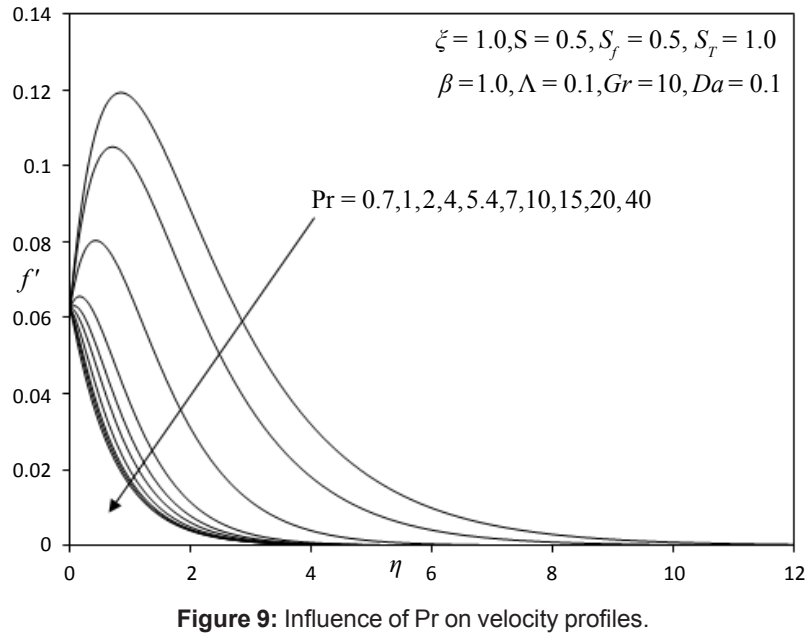

$\xi$ is extremely important since in two extreme cases it corresponds to stagnation-point flows. For $\xi \sim 0$, the location is in the vicinity of 


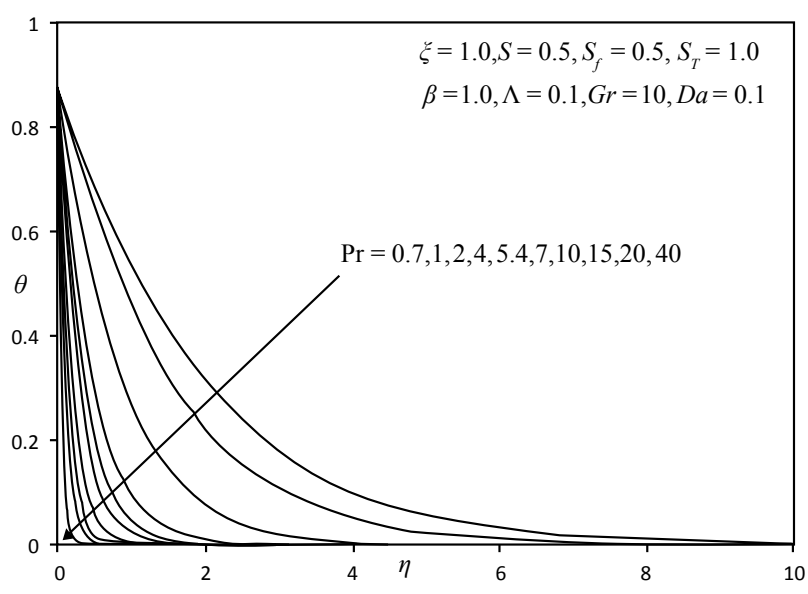

Figure 10: Influence of Pr on temperature profiles.

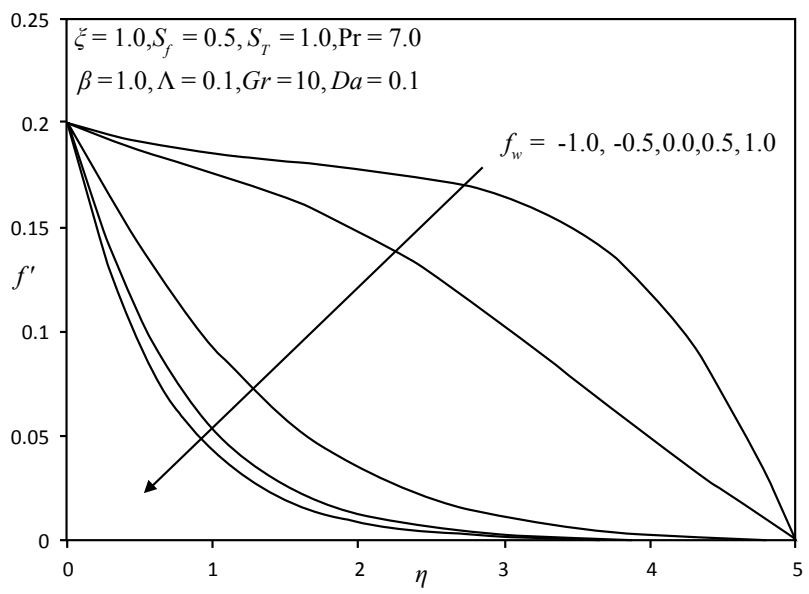

Figure 11: Influence of $f_{w}$ on velocity profiles

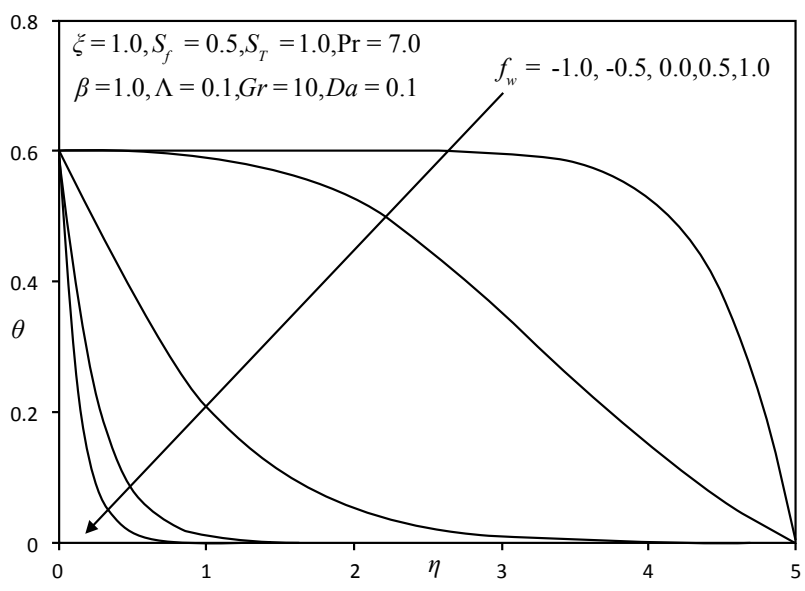

Figure 12: Influence of $f_{w}$ on temperature profiles.

the lower stagnation point on the sphere. The governing dimensionless equations (8) to (9) in this case reduce to the following ordinary differential equations:

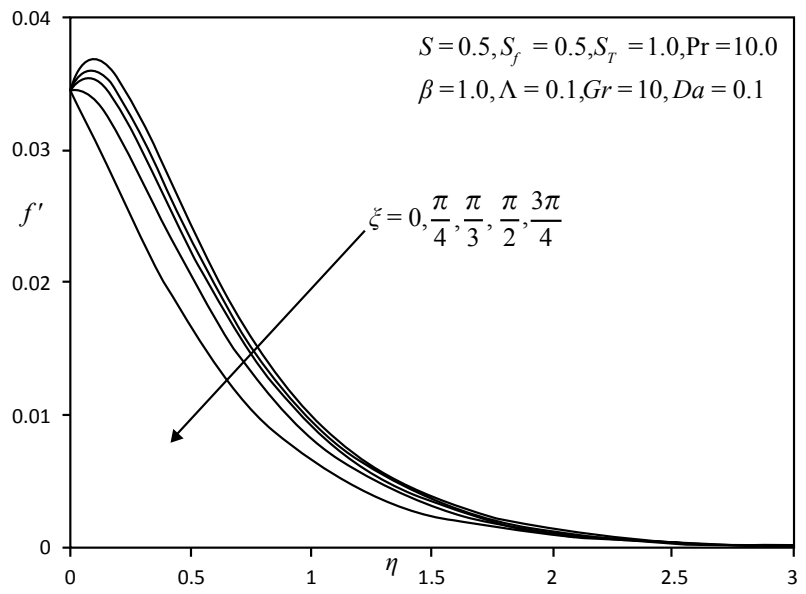

Figure 13: Influence of $\xi$ on velocity profiles.

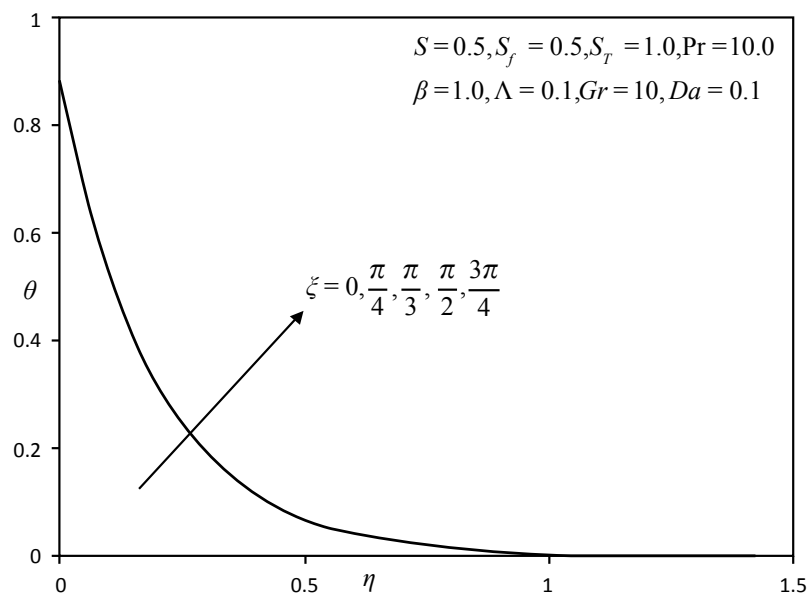

Figure 14: Influence of $\xi$ on temperature profiles.

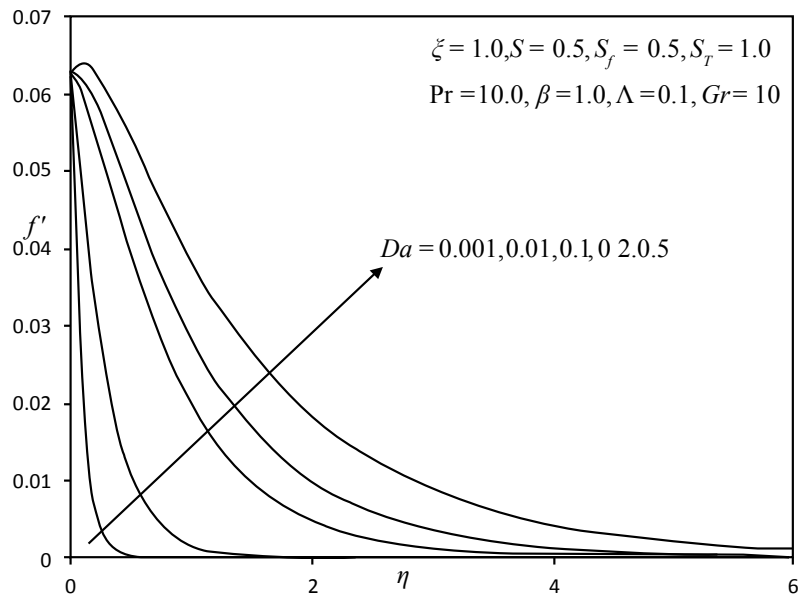

Figure 15: Influence of $\mathrm{Da}$ on velocity profiles.

$\left(1+\frac{1}{\beta}\right) v^{\prime}+f v-u^{2}-\left(\frac{1}{D a G r^{1 / 2}}\right) u+\theta=0$ 


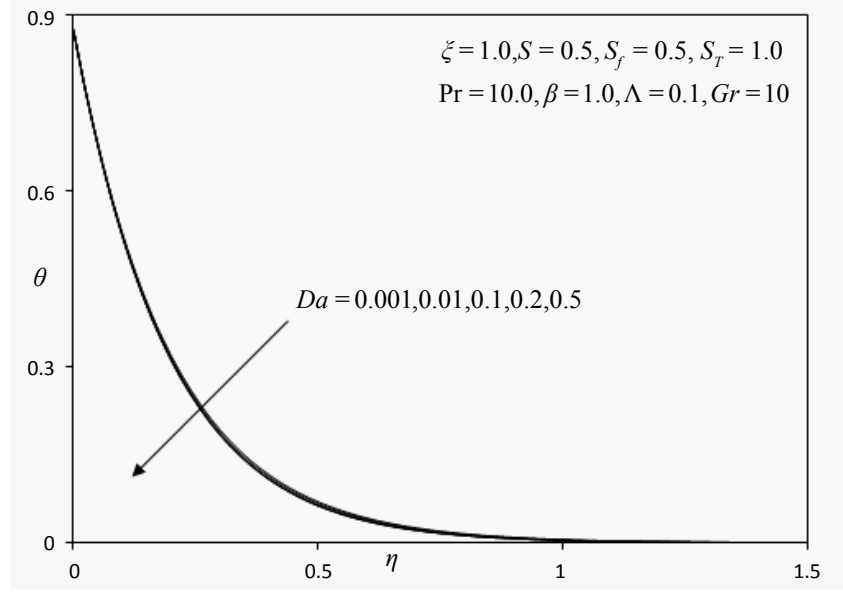

Figure 16: Influence of Da on temperature profiles.

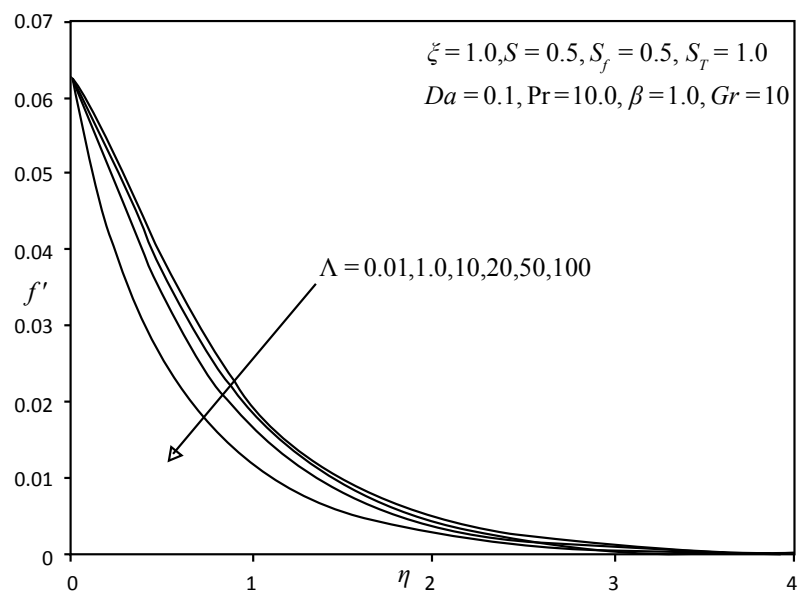

Figure 17: Influence of $\Lambda$ on velocity profiles.

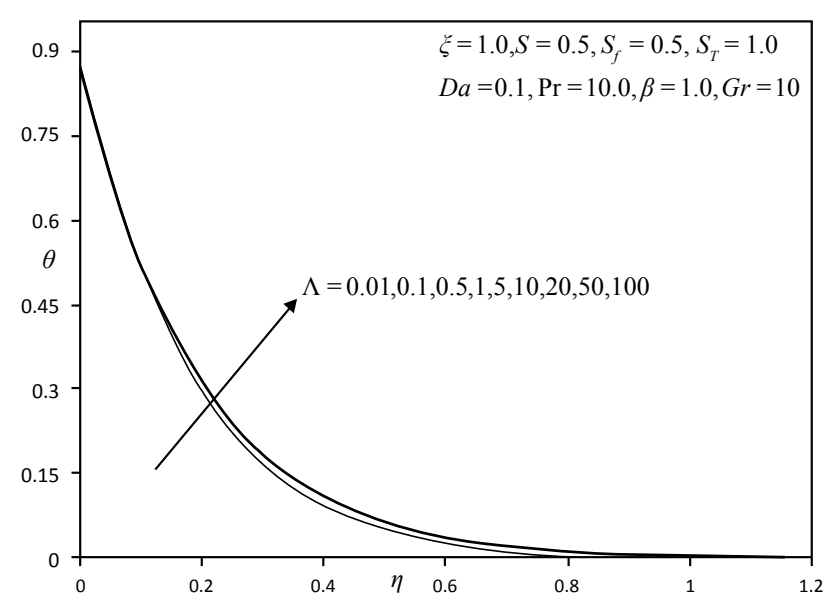

Figure 18: Influence of $\Lambda$ on temperature profiles.

$$
\frac{1}{\operatorname{Pr}} t^{\prime}+f t=0
$$

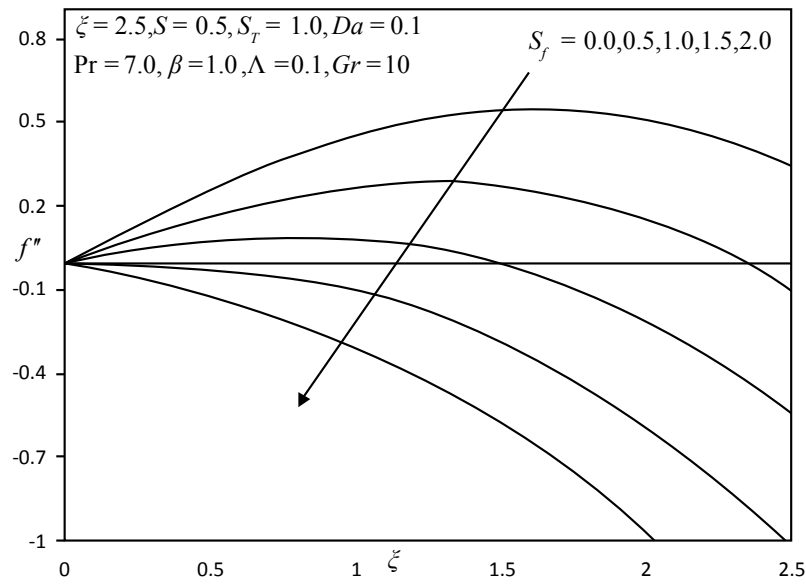

Figure 19: Effect of $S_{f}$ on the Skin-friction Coefficient results.

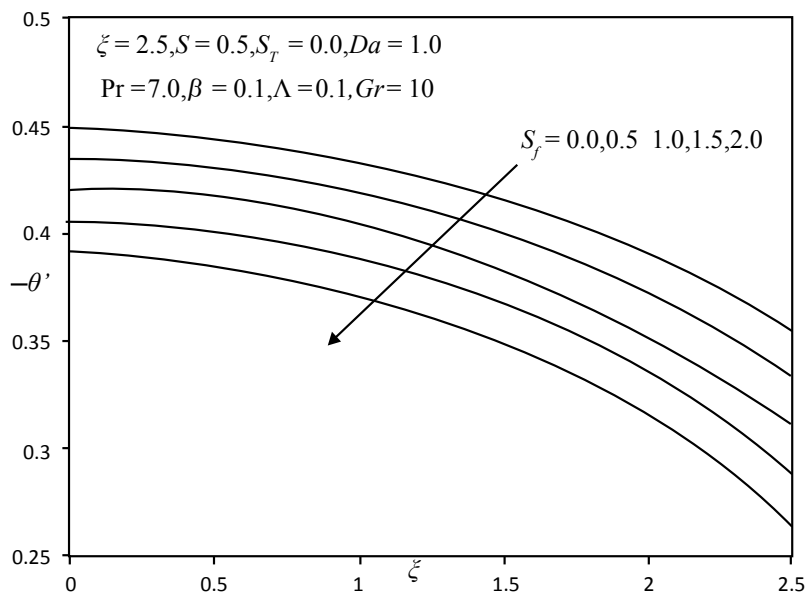

Figure 20: Effect of $S_{f}$ on the local Nusselt number results.

Since $\frac{\sin \xi}{\xi} \rightarrow 0 / 0$ i.e. 1 , so that $\frac{\sin \xi}{\xi} \theta \rightarrow \theta$; The extreme case is $\xi$ $\sim \pi$, which physically corresponds to the upper stagnation point on the sphere surface (diametrically opposite to the lower stagnation point).

In figures 3 and 4 the influence of velocity slip parameter on velocity and temperature distributions is illustrated. Dimensionless velocity component (Figure 3 ) at the wall is strongly reduced with an increase in slip parameter $S_{f}$. There will be a corresponding decrease in the momentum (velocity) boundary layer thickness. The influence of $S_{f}$ is evidently more pronounced closer to the cylinder surface $(\eta=0)$. Further from the surface, there is a transition in velocity slip effect, and the flow is found to be accelerated markedly. Smooth decays of the velocity profiles are observed into the free stream demonstrating excellent convergence of the numerical solution. These trends in the response of velocity field in external thermal convection from a cylinder were also observed by Wang and Ang [35] and Wang [36]. Furthermore the acceleration near the wall with increasing velocity slip effect has been computed by Crane and McVeigh [37] using asymptotic methods, as has the retardation in flow further from the wall. The switch in velocity slip effect on velocity evolution has also been observed for the case of a power-law rheological fluid by Ojadi et al. [38]. Figure 4 indicates that an increase in velocity slip parameter significantly enhances temperature in the flow field and 


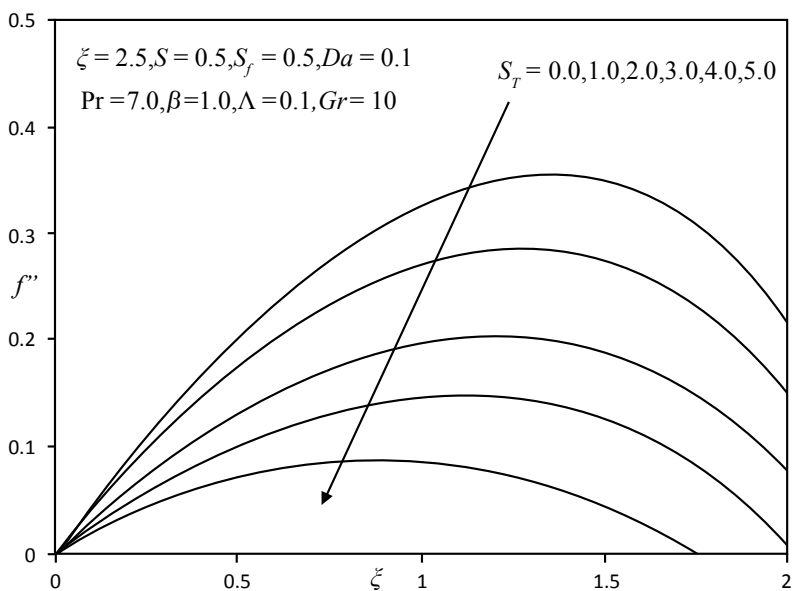

Figure 21: Effect of $S_{T}$ on the Skin-friction Coefficient results.

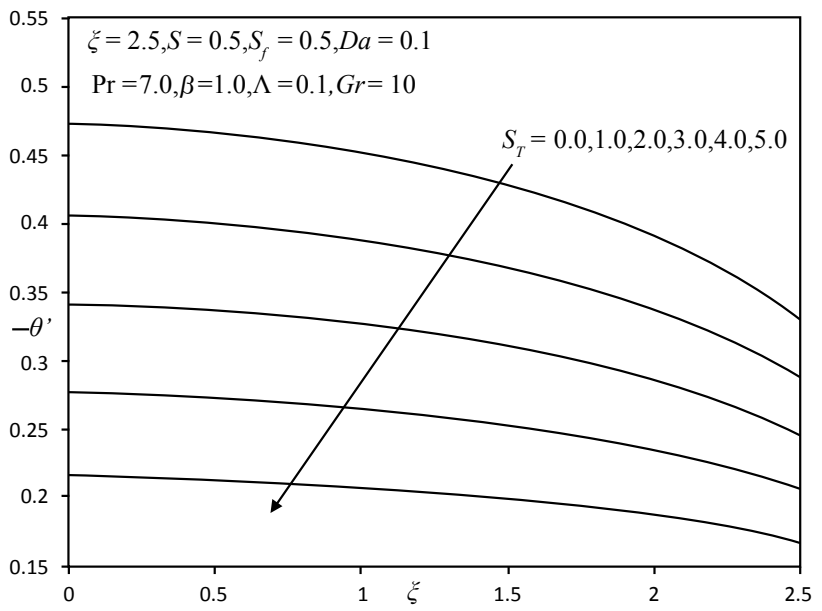

Figure 22: Effect of $S_{T}$ on the local Nusselt number results.

thereby increases thermal boundary layer thickness enhances. This will result therefore in the transport of more thermal energy from the cylinder surface to the Casson fluid and will therefore accentuate heat transfer to the fluid, as noted also by Wang [36]. Temperature profiles consistently decay monotonically from a maximum at the cylinder surface to the free stream. All profiles converge at large value of radial coordinate, again showing that convergence has been achieved in the numerical computations. A similar pattern of thermal response to that computed in figure 4 . For a wide range of velocity slip parameters has been noted by Aziz [39] who has indicated also that temperature is enhanced since increasing velocity slip parameter decreases shear stresses and this permits a more effective transfer of heat from the wall to the fluid regime.

In figures 5 and 6 the variation of velocity and temperature with the transverse coordinate $(\eta)$, with increasing thermal slip parameter $S_{T}$ is depicted. The response of velocity is much more consistent than for the case of changing velocity slip parameter (Figure 3) it is strongly decreased for all locations in the radial direction. The peak velocity accompanies the case of no thermal slip $\left(S_{T}=0\right)$. The maximum deceleration corresponds to the case of strongest thermal slip $\left(S_{T}=5\right)$. Temperatures (Figure 6) are also strongly depressed with increasing thermal slip. The maximum effect is observed at the wall. Further into the free stream, all temperature profiles converge smoothly to the vanishing value. The numerical computations correlate well with the results of Larrode et al. [40] who also found that temperature is strongly lowered with increasing thermal slip and that this is attributable to the decrease in heat transfer from the wall to the fluid regime, although they considered only a Newtonian fluid.

Figures 7 and 8 depict the influence Casson fluid parameter, $\beta$ on velocity and temperature profiles. This parameter features in the shear term in the momentum boundary layer equation (8), and also in the velocity boundary condition (10). For Newtonian flow, yield stress $\mathrm{p}_{\mathrm{y}}$ is zero and $\beta=\mu_{B} \frac{\sqrt{2 \pi_{c}}}{p_{y}} \rightarrow \infty$ i.e. the appropriate term in eqn. (8) reduces from $\left(1+\frac{1}{\beta}\right) f^{\prime \prime \prime} \rightarrow 1$. Similarly the velocity boundary condition in (10) reduces from $\left(1+\frac{1}{\beta}\right) S_{f} f^{\prime \prime}(0) \rightarrow S_{f} f^{\prime \prime}(0)$. An increase in $\beta$ implies a decrease therefore in yield stress of the Casson fluid. This effectively facilitates flow of the fluid i.e. accelerates the boundary layer flow close to the cylinder surface, as demonstrated by figure 7 . Since the Casson parameter is also present in the wall boundary

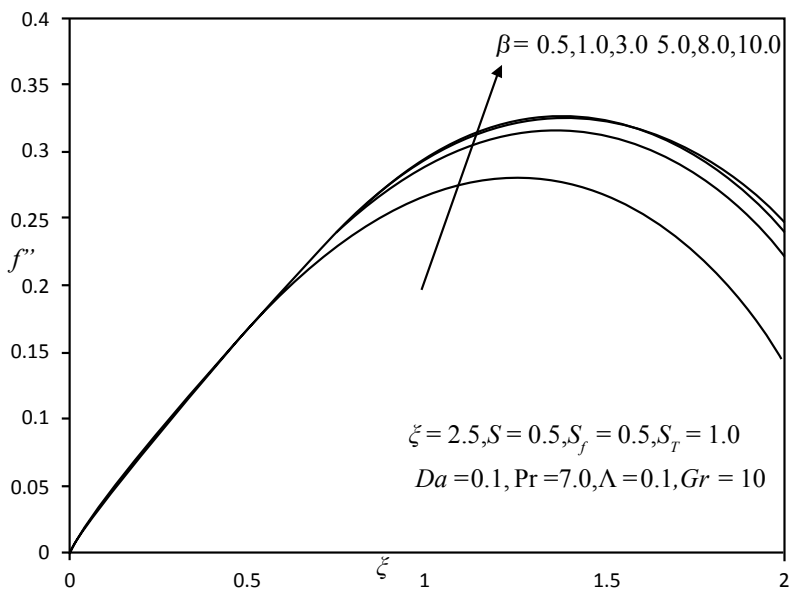

Figure 23: Effect of $\beta$ on the Skin-friction Coefficient results.

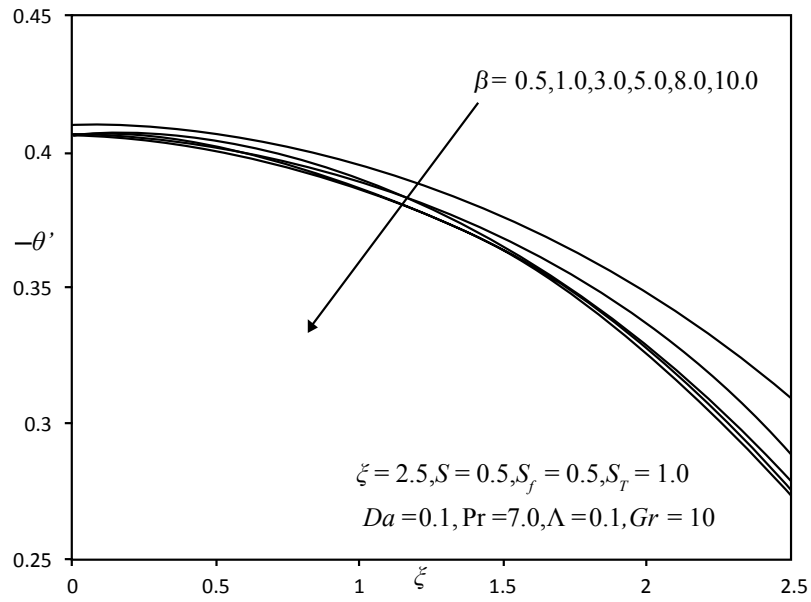

Figure 24: Effect of $\beta$ on the local Nusselt number results. 
condition, the acceleration effect is only confined to the region close to the cylinder surface. Further from this zone, the velocity slip factor, $S_{f}$ will exert a progressively reduced effect and an increase in Casson parameter, $\beta$, will manifest with a deceleration in the flow. Overall however the dominant influence of $\beta$, is near the wall and is found to be assistive to momentum development (with larger $\beta$ values the fluid is closer in behaviour to a Newtonian fluid and further departs from plastic flow) Only a very small decrease in temperature is observed with a large enhancement in Casson fluid parameter, as shown in figure 8. The Casson parameter does not arise in the thermal boundary layer equation (9), nor does it feature in the thermal boundary conditions. The influence on temperature field is therefore experienced indirectly via coupling of the thermal equation (9) with the momentum equation (8). Similar behaviour to the computations shown in figures 7 and 8 has been observed by Attia and Sayed-Ahmed [41] who also observed acceleration in Casson fluid flow near a curved surface, and additionally by Mustafa et al. [42] who also observed an elevation in velocities near the wall and a slight reduction in temperatures throughout the boundary layer regime.

Figures 9 and 10 present the effect of Prandtl number (Pr) on the velocity and temperature profiles along the radial direction, normal to the cylinder surface. Prandtl number embodies the ratio of viscous diffusion to thermal diffusion in the boundary layer regime. It also expresses the ratio of the product of specific heat capacity and dynamic viscosity, to the fluid thermal conductivity. When Pr high, viscous diffusion rate is exceeds thermal diffusion rate. An increase in Pr from 0.7 through 1.0, 2.0, 4.0, 5.47.0,10.0,15.0,20.0 to 40.0 is found to significantly depress velocities (Figure 9) and this trend is sustained throughout the regime i.e. for all values of the radial coordinate, $\eta$. For $\operatorname{Pr}<1$, thermal diffusivity exceeds momentum diffusivity i.e. heat will diffuse faster than momentum. Therefore for lower Pr fluids (e.g. $\operatorname{Pr}=0.7$ ), the flow will be accelerates whereas for greater Pr fluids (e.g. $\operatorname{Pr}=1$ ) it will be strongly decelerated, as observed in fig. For $\operatorname{Pr}=1.0$, both the viscous and energy diffusion rates will be the same as will the thermal and velocity boundary layer thicknesses. This case can be representative of food stuffs e.g. low-density polymorphic forms of chocolate suspensions, as noted by Steffe [27] and Debaste et al. [43]. Temperature is found to be strongly reduced with increasing Prandtl number. For larger Pr values, the decay is found to be increasingly monotonic. Therefore for lower thermal conductivity fluids (as typified by liquid chocolate and other foodstuffs), lower temperatures are observed throughout the boundary layer regime.

Figures 11 and 12 illustrate the influence of wall transpiration on the velocity and temperature functions with radial distance $\eta$. With an increase in suction $\left(f_{w}>0\right)$ the velocity is clearly decreased i.e. the flow is decelerated. Increasing suction causes the boundary layer to adhere closer to the flow and destroys momentum transfer; it is therefore an excellent control mechanism for stabilizing the external boundary layer flow on the circular cylinder. Conversely with increased blowing i.e. injection of fluid via the cylinder surface in to the porous medium regime, $\left(f_{w}<0\right)$, the flow is strongly accelerated i.e. velocities are increased. As anticipated the case of a solid cylinder $\left(f_{w}=0\right)$ falls between the weak suction and weak blowing cases. Peak velocity is located, as in the figures described earlier, at close proximity to cylinder surface. With a decrease in blowing and an increase in suction the peaks progressively displace closer to the cylinder surface, a distinct effect described in detail in several studies of non-Newtonian boundary layers [24,44-47]. Temperature $\theta$ is also elevated considerably with increased blowing at the cylinder surface and depressed with increased suction. The temperature profiles, once again assume a continuous decay from the cylinder surface to the free stream, whereas the velocity field initially ascends, peaks and then decays in to the free stream. The strong influence of wall transpiration (i.e. suction or injection) on boundary layer variables is clearly highlighted. Such a mechanism is greatly beneficial in achieving flow control and regulation of heat and mass transfer characteristics in food processing from a cylindrical geometry.

In figures 13 and 14 the variation of velocity and temperature fields with different transverse coordinate, $\xi$, is shown. In the vicinity of the cylinder surface, velocity $\left(f^{\prime}\right)$ is found to be maximized closer to the lower stagnation point and minimized with progressive distance away from it i.e. the flow is decelerated with increasing $\xi$ However further from the wall, this trend is reversed and a slight acceleration in the flow is generated with greater distance from the lower stagnation point i.e. velocity values are higher for greater values of $\xi$, as we approach the upper stagnation point Temperature $\theta$, is found to noticeably increase through the boundary layer with increasing $\xi$ values. Evidently the fluid regime is cooled most efficiently at the lower stagnation point and heated more effectively as we progress around the cylinder periphery upwards towards the upper stagnation point. These patterns computed for temperature and velocity evolution around the cylinder surface are corroborated with many other studies including work on nonNewtonian Casson fluid convection by Kandasamy et al. [48] and studies of Newtonian convection from a cylinder by Wang [36] and Prasad et al. [49].

Figures 15 and 16 depicts the velocity response to a change in Darcy number, $D a$. This parameter is directly proportional to the permeability of the regime and arises in the linear Darcian drag force term in the momentum equation (8), via, $-\frac{f^{\prime}}{D a G r^{1 / 2}}$. As such increasing $D a$ will serve to reduce the Darcian impedance since progressively less fibres will be present adjacent to the cylinder in the porous regime to inhibit the flow. The boundary layer flow will therefore be accelerated and indeed this is verified in figure 15 where we observe a dramatic rise in flow velocity $\left(f^{\prime}\right)$, with an increase in $D a$ from 0.001 through 0.01 , $0.1,0.2$ and 0.5 . In close proximity to the cylinder surface a velocity shoot is generated; with increasing Darcy number this peak migrates slightly away from the wall into the boundary layer. Evidently lower permeability materials serve to decelerate the flow and this can be exploited in materials processing operation where the momentum transfer may require regulation

Figure 17 depicts the velocity $\left(f^{\prime}\right)$ response for different values of Forchheimer inertial drag parameter $(\Lambda)$, with radial coordinate $(\eta)$. The Forchheimer drag force term, $\left(-\xi \Lambda f^{\prime 2}\right)$ in the dimensionless momentum conservation equation (8) is quadratic and with an increase in $\Lambda$ (which is in fact related to the geometry of the porous medium) this drag force will increase correspondingly. As such the impedance offered by the fibres of the porous medium will increase and this will effectively decelerate the flow in the regime, as testified to by the evident decrease in velocities shown in figure 17. The Forchheimer effect serves to super seed the Darcian body force effect at higher velocities, the latter is dominant for lower velocity regimes and is a linear body force. The former is dominated at lower velocities (the square of a low velocity yields an even lower velocity) but becomes increasingly dominant with increasing momentum in the flow i.e. when inertial effects override the viscous effects (Figure 17). 
Figure 18 shows that temperature $\theta$ is increased continuously through the boundary layer with distance from the cylinder surface, with an increase in $\Lambda$, since with flow deceleration, heat will be diffused more effectively via thermal conduction and convection. The boundary layer regime will therefore be warmed with increasing $\Lambda$ and boundary layer thickness will be correspondingly increased, compared with velocity boundary layer thickness, the latter being reduced.

Figures 19 and 20 show the effect of velocity slip parameter $S_{f}$ on cylinder surface shear stress $\left(f^{\prime \prime}\right)$ and local Nusselt number $\left(-\theta^{\prime}\right)$ variation. In consistency with the earlier graphs described for velocity evolution, with an increase in $S_{f}$, wall shear stress is consistently reduced i.e. the flow is decelerated along the cylinder surface. Again this trend has been observed by Wang and Ang [35] and Wang [36] using asymptotic methods. There is also a progressive migration in the peak shear stress locations further from the lower stagnation point, as wall slip parameter is increased. The impact of wall slip is therefore significant on the boundary layer characteristics of Casson flow from a cylinder. With an increasing $S_{f}$, the local Nusselt number is also considerably decreased and profiles are generally monotonic decays. Maximum local Nusselt number always arises at the cylinder surface and is minimized with proximity to the lower stagnation point i.e. greater distance from the upper stagnation point. This pattern of behaviour has also been observed and emphasized by Yih [29] for Newtonian flow. In both figures 19 and 20, skin friction coefficient and local Nusselt number are maximized for the case of no-slip i.e. $S_{f}=0$, this result concurring with the analyses of Wang [36] and also Hayat et al. [47].

Figures 21 and 22 show the effect of thermal slip parameter $S_{T}$ on dimensionless wall shear stress function i.e. skin friction coefficient and local Nusselt number, respectively. Increasing $S_{T}$ is found to decrease both skin friction coefficient and local Nusselt number. A similar set of profiles is computed as in figure 21 for velocity distributions, and we observe that with increasing thermal slip, peak velocities are displaced closer to the lower stagnation point. For lower values of thermal slip, the plots are also similar to those in figure 22, and have a parabolic nature; however with $S_{T}$ values greater than 1, the profiles lose their curvature and become increasingly linear in nature. This trend is maximized for the highest value of $S_{T}(=5.0)$ for which local Nusselt number is found to be almost invariant with transverse coordinate, $\xi$.

Figures 23 and 24 illustrate the effect of Casson fluid parameter, $\beta$, on skin friction coefficient and local Nusselt number, respectively. With an increase in $\beta$ the skin friction coefficient increases, since as computed earlier, the flow velocity is enhanced with higher values of $\beta$. Larger $\beta$ values correspond to a progressive decrease in yield stress of the Casson fluid i.e. a reduction in rheological characteristics. With higher $\beta$ the flow approaches closer to Newtonian behaviour and the fluid is able to shear faster along the cylinder surface. Local Nusselt number is conversely found to decrease slightly as Casson fluid parameter is increased. This concurs with the earlier computation (Figure 8) on temperature distribution. With increasing $\beta$ values, less heat is transferred from the cylinder surface to the fluid regime, resulting in lower temperatures in the regime external to the cylinder and lower local Nusselt numbers, as observed in figure 24 .

\section{Conclusions}

Numerical solutions have been presented for steady flow and heat transfer of Casson fluid from a permeable horizontal circular cylinder with partial slip in a Non-Darcy porous medium. A Robust, validated implicit finite difference scheme has been employed. The results in summary have shown that,

1. Increasing the velocity slip parameter, $S_{f}$, reduces the velocity near the cylinder surface and also skin friction coefficient and also increases temperature and decreases local Nusselt number.

2. Increasing the thermal slip parameter, $S_{T}$, decreases velocity and skin friction coefficient and also reduces temperature for all values of radial coordinate i.e. throughout the boundary layer regime, and furthermore decreases local Nusselt number.

3. Increasing the Casson fluid parameter, $\beta$, increases the velocity near the cylinder surface but decreases velocity further from the cylinder, and also fractionally lowers the temperature throughout the boundary layer regime.

4. Increasing the Casson fluid parameter, $\beta$, strongly increases the wall shear stress (skin friction coefficient) and slightly decreases the local Nusselt number, with the latter more significantly affected at large distances from the lower stagnation point i.e. higher values of transverse coordinate.

5. Increasing Prandtl number, Pr, decelerates the flow and also strongly depresses temperatures, throughout the boundary layer regime.

6. Increasing suction at the cylinder surface $\left(f_{w}>0\right)$ decelerates the flow whereas increasing injection $\left(f_{w}<0\right.$, i.e. blowing $)$ induces a strong acceleration.

7. Increasing suction at the cylinder surface $\left(f_{w}>0\right)$ reduces temperature whereas increasing injection $\left(f_{w}<0\right.$ i.e. blowing $)$ induces the opposite response and elevates temperature.

8. Increasing transverse coordinate, $\xi$, depresses velocity near the cylinder surface but enhances velocity further from the cylinder whereas it continuously increases temperature throughout the boundary layer.

9. Increasing Darcy number $(D a)$, velocity increases but reduces the temperature.

10. Increasing Forchheimer inertial drag parameter $(\Lambda)$ reduces velocity but elevates temperature.

The current study has been confined to steady-state flow i.e. ignored transient effects [50] and also neglected thermal radiation heat transfer effects $[51,49]$. These aspects are also of relevance to rheological food processing simulations and will be considered in future investigations.

\section{Acknowledgements}

The authors are grateful to both reviewers for their constructive comments which have helped to improve the present article.

\section{References}

1. Schowalter WR (1978) Mechanics of Non-Newtonian Fluids. Pergamon Press, USA.

2. Jamil M, Fetecau C, Imran M (2011) Unsteady helical flows of Oldroyd-B fluids Commu Nonlinear Sci Num Simu 16: 1378-1386.

3. Nazar M, Fetecau C, Vieru D, Fetecau C (2010) New exact solutions 
Citation: Ramachandra Prasad V, Subba Rao A, Anwar Bég O (2013) Flow and Heat Transfer of Casson Fluid from a horizontal Circular Cylinder with Partial Slip in non-Darcy porous Medium. J Appl Computat Math 2: 127. doi:10.4172/2168-9679.1000127

Page 11 of 12

corresponding to the second problem of Stokes for second grade fluids. Nonlinear Analysis: Real World Appl 11: 584-591.

4. Fetecau C, Hayat T, Zierep J, Sajid M (2011) Energetic balance for the Rayleigh-Stokes problem of an Oldroyd-B fluid. Nonlinear Analysis: Real World Appl. 12: 1-13

5. Wang SW, Tan WC, (2008) Stability analysis of double-diffusive convection of Maxwell fluid in a porous medium heated from below. Phys Lett A 372: 30463050.

6. Tan WC, Xu MY (2004) Unsteady flows of a generalized second grade fluid with the fractional derivative model between two parallel plates. Acta Mech Sin 20: 471-476

7. Zhang ZY, Fu CJ, Tan WC, Wang CY (2007) On set of oscillatory convection in a porous cylinder saturated with a viscoelastic fluid. Phys Fluids 19

8. Rashidi MM, Chamkha AJ, Keimanesh M (2011) Application of multi-step differential transform method on flow of a second grade fluid over a stretching or shrinking sheet. American J Comput Math 6: 119-128.

9. Ali N, Hayat T, Asghar S (2009) Peristaltic flow of Maxwell fluid in a channel with compliant walls, Chaos, Solitons\& Fractals 39: 407-416.

10. Hayat T, Qasim M, Abbas Zand, Hendi AA (2010) Magneto hydrodynamic flow and mass transfer of a Jeffery fluid over a nonlinear stretching surface. $Z$ Naturforsch A 64: 1111-1120.

11. Hussain M, Hayat T, Asghar S, Fetecau C (2010) Oscillatory flows of second grade fluid in a porous spac, Nonlinear Analysis: Real World Appl 11: 24032414.

12. Dorfman, KD, Brenner $\mathrm{H}$ (2002) Generalized Taylor-Aris dispersion in discrete spatially periodic networks: Microfluidic applications. Phys Rev E 65: 20-37.

13. Delgado JMPQ (2007) 'Mass Transfer from a Plane Surface Immersed in a Porous Medium with a Moving Fluid'. Chemical Engineering Research and Design 85: 386-394.

14. Minkin L (2003) Thermal diffusion of radon in porous media. Radiation Protection Dosimetry 106: 267-272.

15. Seo T, Kim HD, Choi JH, Chung JH (1998) Mathematical modeling of flow field in ceramic candle filter. J. Thermal Science. $7 ;$ 85-88

16. Saffar Al, Ozturk B, Hughes R (1997) 'A Comparison of porous and non-porous gas-liquid membrane contactors for gas separation'. Chemical Engineering Research and Design 75: 685-692.

17. Ledvinkova B, Keller F, Kosek J, and Nieken U (2008) Mathematical modeling of the generation of the secondary porous structure in a monolithic adsorbent. Chem Eng J 140: 578-585.

18. Turner IW, Puiggali JR, Jomaa W (1998) A numerical investigation of combined microwave and convective drying of a hygroscopic porous material: a study based on pine wood. Chemical Engineering Research and Design 76: 193-209.

19. Pomès V, Fernández A, Houi D (2002) Characteristic time determination for transport phenomena during the electrokinetic treatment of a porous medium Chem Eng J 87: 251-260.

20. Islam MR (1993) Route to chaos in chemically enhanced thermal convection in porous media. Chemical Engineering Communications 124: 77-95.

21. Albusairi B, Hsu JT (2004) Flow through beds of perfusive particles: effective medium model for velocity prediction within the perfusive media. Chem Eng $J$ 100: 79-84.

22. Khachatoorian R, Yen TF (2005) Numerical modeling of in situ gelation of biopolymers in porous media. J Petroleum Science and Engineering 48: 161 168.

23. Casson N (1959) In Reheology of Dipersed system. Peragamon press, Oxford.

24. Nakamura M, Sawada T (1988) Numerical study on the flow of a non-Newtonian fluid through an axisymmetric stenosis. J Biomechanical Eng 110: 137-143.

25. Bird RB, Dai GC, Yarusso BJ (1983) Rheology and flow of viscoplastic materials. Rev Chem Eng 1: 1-70.

26. Derek C, Tretheway DC, Meinhart CD (2002) Apparent fluid Slipathydrophobic microchannel walls. Phy Fluids 14: 1-9.

27. Mustafa M, Hayat T, Pop I, Aziz A (2011) "Unsteady boundary layer flow of a
Casson fluid due to an impulsively started moving flat plate". Heat Transfer Asian Res 40: 563-576

28. Nadeem S, RizwanUI Haq, Lee C (2012) MHD flow of a Casson fluid over an exponentially shrinking sheet. Scientia Inertica Transactions B : Mechanical Engineering 19: 1550-1553.

29. Yih KA (2000) Effect of blowing/suction on MHD-natural convection over horizontal cylinder: UWT or UHF, Acta Mechanica 144: 17-27.

30. Keller HB, (1970) A new difference method for parabolic problems. Numerical Methods for Partial Differential Equations.

31. Ramachandra Prasd V, Vasu B, Anwer Beg O (2011) 'Thermo-Diffusion and Diffusion-Thermo effects on boundary layer flows'. LAP LAMBERT Academic Publishing GmbH \&co KG, Saarbrucken, Germany.

32. Ramachandra Prasd V, Vasu B, Anwer Beg O (2011) 'Thermo-diffusion and diffusion-thermo effects on MHD free convection flow pasta vertical porous plate embedded in a non-Darcian porous medium'. Chemical Engineering Journal 173: 598-606.

33. Anwar Bég O, Ramachandra Prasad V, Vasu B, Bhaskar Reddy N, Li Q et al. (2011) Free convection heat and mass transfer from an isothermal sphere to a micropolarregime with Soret/Dufour effects. International Journal of Heat and Mass Transfer' 54: 9-18.

34. Cebeci T, Bradshaw P (1984) Physical and Computational Aspects of Convective Heat Transfer. Springer, USA.

35. Wang CY, Chiu On Ng (2011) Slip flow due to a stretching cylinder, Int J NonLinear Mechanics 46:1191-1194.

36. Wang CY (2007) Stagnation flow on a cylinder with partial slip-an exact solution of the Navier-Stokes equations, IMA J Applied Mathematics 72: 271-277.

37. Crane LJ, McVeigh AG (2010) Uniform slip flow on a cylinder. PAMM: Proc Appl Math Mech 10: 477-478.

38. Ajadi SO, Adegoke A, Aziz A (2009) Slip boundary layer flow of non-Newtonian fluid over a flat plate with convective thermal boundary condition. Int J Nonlinea Science 8: 300-306.

39. Aziz A (2010) Hydrodynamic and thermal slip flow boundary layers over a flat plate with constant heat flux boundary condition. Commun Nonlinear Sci 15 573-580.

40. Larrode FE, Housiadas C, Drossinos Y (2000) Slip-flow heat transfer in circular tubes. Int J Heat Mass Transfer 43: 2669-2680.

41. Attia H, Sayed-Ahmed ME (2010) Transient MHD Couette flow of a Casson fluid between parallel plates with heat transfer. Italian J Pure Applied Mathematics 27: 19-38.

42. Mustafa M, Hayat T, Pop I, Aziz A (2011) Unsteady boundary layer flow of a Casson fluid due to an impulsively started moving flat plate. Heat TransferAsian Research 40: 563-576.

43. Debaste F, Kegelaers Y, Ben Hamor H, Halloin V (2007) Contribution to the modelling of chocolate tempering. Proc European Congress of Chemical Engineering (ECCE-6), Copenhagen, Denmark, 16-20.

44. Anwar Bég O, Abdel Malleque K, Islam MN (2012) Modelling of OstwalddeWaele non-Newtonian flow over a rotating disk in a non-Darcian porous medium. Int J Applied Mathematics and Mechanics 8: 46-67.

45. GouseMohiddin S, Prasad VR, Anwar Bég O (2010) Numerical study of unsteady free convective heat and mass transfer in a Walters-B viscoelastic flow along a vertical cone. Int J Applied Mathematics and Mechanics 6: 88-114.

46. Rashidi MM, Anwar Bég O, and Rastegari MT (2012) A study of non-Newtonian flow and heat transfer over a non-isothermal wedge using the Homotopy Analysis Method. Chemical Engineering Communications 199: 231-256.

47. Mustafa M, Hayat T, Pop I, Hendi AA (2012) Stagnation-point flow and hea transfer of a Casson fluid towards a stretching sheet. Zeit Fr Natur 67: 70-76.

48. Kandasamy A, Karthik K, Phanidhar PV (2007) Entrance region flow hea transfer in concentric annuli for a Casson fluid, Int Conf Thermal Issues in Emerging Technologies, Theory and Application (ThETA), Cairo, Egypt, January $3^{\text {rd }}-6^{\text {th }}$.

49. Prasad VR, Vasu B, Prashad R, Anwar Bég O (2012) Thermal Radiation Effects on Magnetohydrodynamic Heat And Mass Transfer From a Horizontal Cylinder In A Variable Porosity Regime. J Porous Media 15: 261-281. 
Citation: Ramachandra Prasad V, Subba Rao A, Anwar Bég O (2013) Flow and Heat Transfer of Casson Fluid from a horizontal Circular Cylinder with Partial Slip in non-Darcy porous Medium. J Appl Computat Math 2: 127. doi:10.4172/2168-9679.1000127

50. Prasad VR, Vasu B, Anwar Bég O, Parshad R (2011) Unsteady free convection heat and mass transfer in a Walters-B viscoelastic flow past a semi-infinite vertical plate: a numerical study. Thermal Science 15: S291-S305
51. Prasad VR, Vasu B, Anwar Bég O, Parshad DR (2012) Thermal radiation effects on magneto hydrodynamic free convection heat and mass transfer from a sphere in a variable porosity regime. Commun Nonlinear Sci 17: 654-671. 